\title{
Slicing Strategies for the Generalised Type-2 Mamdani Fuzzy Inferencing System
}

\author{
Sarah Greenfield ${ }^{1}$ and Francisco Chiclana ${ }^{2}$ \\ 1 De Montfort University, Leicester, LE1 9BH, UK, \\ s.greenfield@dmu.ac.uk, \\ WWW home page: http://www.tech.dmu.ac.uk/ sarahg/ \\ 2 De Montfort University, Leicester, LE1 9BH, UK.
}

\begin{abstract}
As a three-dimensional object, there are a number of ways of slicing a generalised type- 2 fuzzy set. In the context of the Mamdani Fuzzy Inferencing System, this paper concerns three accepted slicing strategies, the vertical slice, the wavy slice, and the horizontal slice or $\alpha$-plane. Two ways of defining the generalised type- 2 fuzzy set, vertical slices and wavy slices, are presented. Fuzzification and inferencing is presented in terms of vertical slices. After that, the application of all three slicing strategies to defuzzification is described, and their strengths and weaknesses assessed.
\end{abstract}

Keywords: type-2 fuzzy set, defuzzification, type-reduction, Mamdani Fuzzy Inferencing System

\section{Introduction}

Type-2 fuzzy sets are an extension of type-1 fuzzy sets in which the sets' membership grades are type-1 fuzzy sets. The concept dates back to Zadeh's seminal paper of 1975 [30. They take two forms, the interval, for which all secondary membership grades are 1, and the generalised, where the secondary membership grade may take any value between 0 and 1 . For the computationally simpler interval type-2 Fuzzy Inferencing System (FIS) 24 applications in areas such as control, simulation and optimisation have been developed [1 6. So far, generalised type-2 fuzzy applications are few in number [18, 20, 24]. This is attributable to the enormous computational complexity of generalised type-2 fuzzy inferencing. Strategies have been developed that reduce the computational complexity of all stages of the generalised type-2 FIS [14, 15, 22, 33, and of particular relevance to this paper, 21. In 11 three of these strategies are evaluated.

Uncertainty is ineradicably present in the factors upon which decisions are made. The ability to deal with uncertainty is desirable in an FIS because better uncertainty handling gives more accurate outputs. The interval type-2 fuzzy set, an enhancement of the ubiquitous type-1 fuzzy set, has an inbuilt facility to handle uncertain inputs. However the generalised type-2 fuzzy set, an augmentation of the interval type-2 fuzzy set, provides uncertainty handling that is subtle and sophisticated [16. More generalised type-2 applications are desirable, since at 
present the generalised type-2 fuzzy set's remarkable facility for dealing with uncertainty is not being fully exploited (Subsection 5.1).

The focus of this paper is the Mamdani FIS (Fig. 1), in which a crisp numerical input passes through three stages of processing: fuzzification, inferencing, and lastly, the crucial stage of defuzzification. Through defuzzification, the aggregated set produced during the inferencing stage is converted into a crisp number which is the output of the FIS. For discretised type- 1 fuzzy sets, defuzzification is a simple procedure, with several defuzzification techniques available including the centroid, centre of maxima and mean of maxima [19. In contrast, defuzzification of a discretised type-2 fuzzy set (as formed in a type-2 FIS) is a process consisting of two stages [23]:

1. Type-reduction, which converts a type-2 fuzzy set to a type-1 fuzzy set known as the Type-Reduced Set (TRS), and

2. defuzzification of the type-1 TRS.

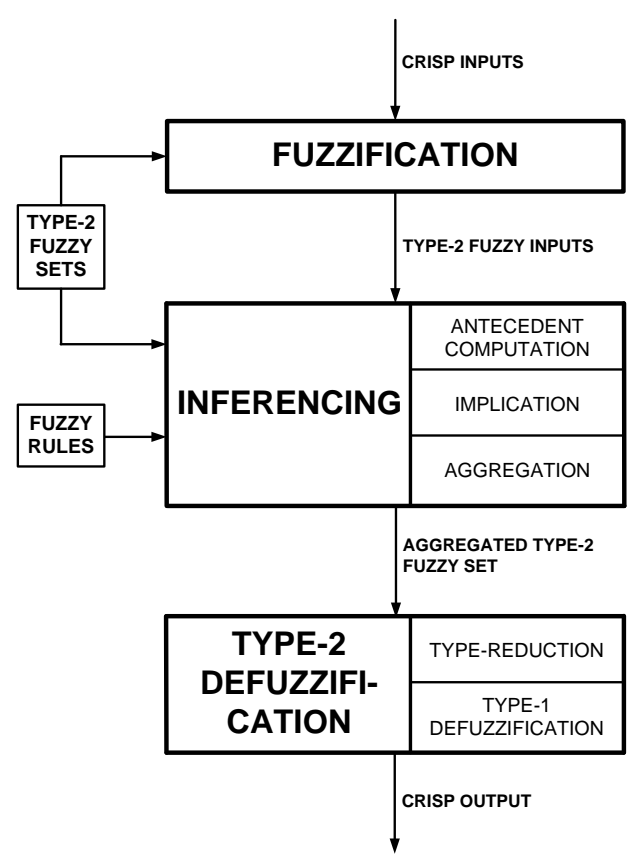

Fig. 1. The Mamdani Type-2 FIS.

\footnotetext{
3 The alternative is the Takagi-Sugeno-Kang FIS for which the output membership functions are either linear or constant; defuzzification is superfluous as the outputs may be aggregated via a simple weighted sum.
} 
The paper is structured as follows: The next section presents two ways of defining the generalised type-2 fuzzy set (vertical slices and wavy slices). Section 3 describes the join and meet inferencing algorithms which employ vertical slices. Section 4 concerns type-2 defuzzification approaches based on wavy slices, vertical slices and horizontal slices ( $\alpha$-planes). Lastly, Sect. 5 concludes the paper.

\section{Defining the Type-2 Fuzzy Set}

This section describes how the type-2 fuzzy set may be defined through either vertical slices or wavy slices.

\subsection{The Vertical Representation}

Let $X$ be a universe of discourse. A type- 1 fuzzy set $A$ on $X$ is characterised by a membership function $\mu_{A}: X \rightarrow[0,1]$ and can be expressed as follows [29]:

$$
A=\left\{\left(x, \mu_{A}(x)\right) \mid \mu_{A}(x) \in[0,1] \forall x \in X\right\}
$$

Let $\tilde{P}(U)$ be the set of fuzzy sets in $U$. A type-2 fuzzy set $\tilde{A}$ in $X$ is a fuzzy set whose membership grades are themselves fuzzy 30 32]. This implies that $\mu_{\tilde{A}}(x)$ is a fuzzy set in $U$ for all $x$, i.e. $\mu_{\tilde{A}}: X \rightarrow \tilde{P}(U)$ and

$$
\tilde{A}=\left\{\left(x, \mu_{\tilde{A}}(x)\right) \mid \mu_{\tilde{A}}(x) \in \tilde{P}(U) \forall x \in X\right\} .
$$

It follows that $\forall x \in X \exists J_{x} \subseteq U$ such that $\mu_{\tilde{A}}(x): J_{x} \rightarrow U$. Applying (1) gives:

$$
\mu_{\tilde{A}}(x)=\left\{\left(u, \mu_{\tilde{A}}(x)(u)\right) \mid \mu_{\tilde{A}}(x)(u) \in U, \forall u \in J_{x} \subseteq U\right\} .
$$

$X$ is called the primary domain and $J_{x}$ the primary membership of $x$ while $U$ is known as the secondary domain and $\mu_{\tilde{A}}(x)$ the secondary membership of $x$. Putting (2) and (3) together we obtain

$$
\tilde{A}=\left\{\left(x,\left(u, \mu_{\tilde{A}}(x)(u)\right)\right) \mid \mu_{\tilde{A}}(x)(u) \in U, \forall x \in X \wedge \forall u \in J_{x} \subseteq U\right\} .
$$

Definition 1 (Vertical Slice [24]). A vertical slice of a type-2 fuzzy set is a plane through the $x$-axis, parallel to the $u-z$ plane.

\subsection{The Wavy Slice Representation Theorem}

An embedded type-2 fuzzy set (embedded set) or wavy slice [11, 24] (Fig. 2) is a special kind of type-2 fuzzy set, which relates to the type- 2 fuzzy set in which it is embedded in this way: For every primary domain value, $x$, there is a unique secondary domain value, $u$, plus the associated secondary membership grade that is determined by the primary and secondary domain values, $\mu_{\tilde{A}}(x)(u)$. 


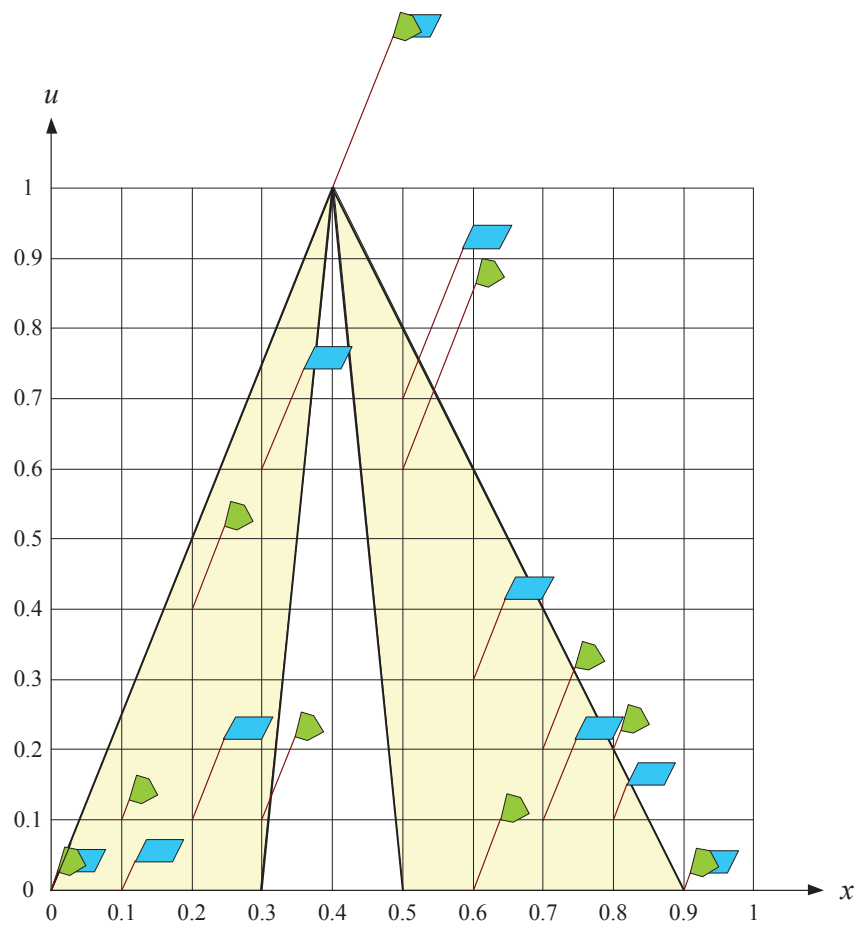

Fig. 2. Two embedded type-2 fuzzy sets, indicated by different flag styles. The flag position in the $x-u$ plane shows the primary membership grade. The flag height indicates the secondary membership grade.

Definition 2 (Embedded Set). Let $\tilde{A}$ be a type-2 fuzzy set in $X$. For discrete universes of discourse $X$ and $U$, an embedded type- 2 set $\tilde{A}_{e}$ of $\tilde{A}$ is defined as the following type-2 fuzzy set

$$
\tilde{A}_{e}=\left\{\left(x_{i},\left(u_{i}, \mu_{\tilde{A}}\left(x_{i}\right)\left(u_{i}\right)\right)\right) \mid \forall i \in\{1, \ldots, N\}: x_{i} \in X \quad u_{i} \in J_{x_{i}} \subseteq U\right\} .
$$

$\tilde{A}_{e}$ contains exactly one element from $J_{x_{1}}, J_{x_{2}}, \ldots, J_{x_{N}}$, namely $u_{1}, u_{2}, \ldots$, $u_{N}$, each with its associated secondary grade, namely $\mu_{\tilde{A}}\left(x_{1}\right)\left(u_{1}\right), \mu_{\tilde{A}}\left(x_{2}\right)\left(u_{2}\right)$, $\ldots, \mu_{\tilde{A}}\left(x_{N}\right)\left(u_{N}\right)$.

Mendel and John have demonstrated that a type-2 fuzzy set is definable as the union of its embedded type-2 fuzzy sets [11, 24]. This result is known as the type-2 fuzzy set Representation Theorem or wavy slice Representation Theorem, and is formally stated thus [24, Page 121]:

Let $\tilde{A}_{e}^{j}$ denote the $j$ th type- 2 embedded type-2 fuzzy set for type- 2 fuzzy set $\tilde{A}$, i.e.,

$$
\tilde{A}_{e}^{j} \equiv\left\{\left(x_{i},\left(u_{i}^{j}, \mu_{\tilde{A}}\left(x_{i}\right)\left(u_{i}^{j}\right)\right)\right), i=1, \ldots, N\right\}
$$


where $\left\{u_{i}^{j}, \ldots, u_{N}^{j}\right\} \in J_{x_{i}}$. Then $\tilde{A}$ may be represented as the union of its embedded type-2 fuzzy sets, i.e.,

$$
\tilde{A}=\sum_{j=1}^{n} \tilde{A}_{e}^{j}, \text { where } n \equiv \prod_{i=1}^{N} M_{i} .
$$

\section{Type-2 Fuzzy Inferencing using Vertical Slices}

In this section the join and meet algorithms for fuzzification of, and inferencing with, discretised generalised type-2 fuzzy sets are presented [23]. $\tilde{B}$ is

The formula for the join operation of two discretised type- 2 fuzzy sets $\tilde{A}$ and

$$
\mu_{\tilde{A} \cup \tilde{B}}(x)=\sum_{u \in J_{x}^{u}} \sum_{w \in J_{x}^{w}} f_{x}(u) \star g_{x}(w) /(u \vee w) \quad x \in X,
$$

and the formula for the meet operation is

$$
\mu_{\tilde{A} \cap \tilde{B}}(x)=\sum_{u \in J_{x}^{u}} \sum_{w \in J_{x}^{w}} f_{x}(u) \star g_{x}(w) /(u \wedge w) \quad x \in X,
$$

where $\vee$ is the maximum operator, $\wedge$ is the minimum operator, $\star$ signifies a t-norm, and $\sum \sum$ represents union over $J_{x}^{u} \times J_{x}^{w}$.

Join and meet operations proceed vertical slice by vertical sliç so it is sufficient to specify how these operations may be applied to two slices. Let $\tilde{A}$ and $\tilde{B}$ be two type-2 fuzzy sets, in which the co-domains are discretised into $N$ slices, and the domains sliced vertically at the points $x_{\tilde{A}}$ and $x_{\tilde{B}}$ respectively. Two type-1 fuzzy sets,

$$
\begin{aligned}
& S_{\tilde{A}}=\left\{z_{A_{1}} / u_{A_{1}}+z_{A_{2}} / u_{A_{2}}+\cdots+z_{A_{N}} / u_{A_{N}}\right\}, \\
& S_{\tilde{B}}=\left\{z_{B_{1}} / u_{B_{1}}+z_{B_{2}} / u_{B_{2}}+\cdots+z_{B_{N}} / u_{B_{N}}\right\},
\end{aligned}
$$

are generated. To join these two slices necessitates that all $N^{2}$ possible min / max pairings of $S_{\tilde{A}}$ and $S_{\tilde{B}}$ be created: $\min \left(z_{A_{1}}, z_{B_{1}}\right) / \max \left(u_{A_{1}}, u_{B_{1}}\right)+\min \left(z_{A_{1}}, z_{B_{2}}\right) /$ $\max \left(u_{A_{1}}, u_{B_{2}}\right)+\cdots+\min \left(z_{A_{N}}, z_{B_{N}}\right) / \max \left(u_{A_{N}}, u_{B_{N}}\right)$. Similarly, for meet, pairings are generated as follows: $\min \left(z_{A_{1}}, z_{B_{1}}\right) / \min \left(u_{A_{1}}, u_{B_{1}}\right)+\min \left(z_{A_{1}}, z_{B_{2}}\right) /$ $\min \left(u_{A_{1}}, u_{B_{2}}\right)+\cdots+\min \left(z_{A_{N}}, z_{B_{N}}\right) / \min \left(u_{A_{N}}, u_{B_{N}}\right)$.

The next stage is the same for join and meet. For every resultant domain value ('denominator') generated, the maximum membership grade ('numerator') is selected. The resultant set of pairs is the join or meet of the two slices.

\section{Approaches to Type-2 Defuzzification}

This section summarises and evaluates generalised type-2 defuzzification approaches based on wavy slices, vertical slices and horizontal slices.

\footnotetext{
${ }^{4}$ The optimised inferencing algorithms described in [15] employ vertical slices.
} 


\subsection{Exhaustive Defuzzification}

The strategy known as Exhaustive Defuzzification, (so called because every embedded set is processed in turn), is built upon the foundation of the wavy slice Representation Theorem [24] and is therefore precise 5 [24. However it is a very inefficient method owing to its high computational complexity deriving from the large number of embedded sets. Its first and main stage consists of type-reduction of the type-2 fuzzy set to form the TRS [11, defined thus:

Definition 3. The TRS associated with a type-2 fuzzy set $\tilde{A}$ with primary domain $X$ discretised into $N$ points $X=\left\{x_{1}, x_{2}, \ldots, x_{N}\right\}$, is

$$
\begin{aligned}
C_{\tilde{A}}= & \left\{\left(\frac{\sum_{i=1}^{N} x_{i} \cdot u_{k_{i}}}{\sum_{i=1}^{N} u_{k_{i}}}, \mu_{\tilde{A}}\left(x_{1}\right)\left(u_{k_{1}}\right) * \ldots * \mu_{\tilde{A}}\left(x_{N}\right)\left(u_{k_{N}}\right)\right) \mid\right. \\
& \left.\forall\left(u_{k_{1}}, u_{k_{2}}, \ldots, u_{k_{N}}\right) \in J_{x_{1}} \times J_{x_{2}} \times \ldots \times J_{x_{N}} \subseteq U^{N}\right\},
\end{aligned}
$$

where $*$ is a $t$-norm.

Embedded sets (Fig. 2) are referred to implicitly in 8 and explicitly in Algorithm 11.

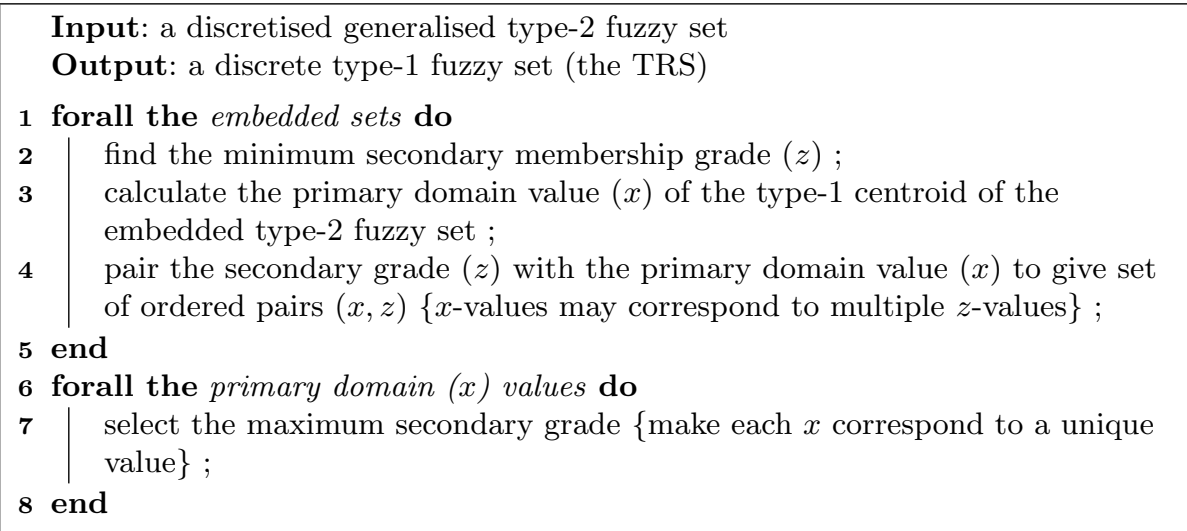

Algorithm 1: Exhaustive type-reduction of a discretised type-2 fuzzy set to a type-1 fuzzy set, adapted from Mendel [23.

\subsection{Vertical Slice Centroid Type-Reduction}

$V S C T R$ is a highly intuitive method employed by John [17]; the paper of Lucas et al. 22] renewed interest in this strategy. In this approach each vertical slice of

\footnotetext{
${ }^{5}$ Discretisation in itself brings an unavoidable element of approximation. However the exhaustive method does not subsequently introduce further inaccuracies.
} 
the type-2 fuzzy set is defuzzified as a type-1 fuzzy set. By pairing the domain value with the defuzzified value of the vertical slice, a type-1 fuzzy set is formed, which is easily defuzzified to give the defuzzified value of the type-2 fuzzy set. Though chronologically preceding it, this method is a generalisation of the NieTan Method for interval type-2 fuzzy sets.

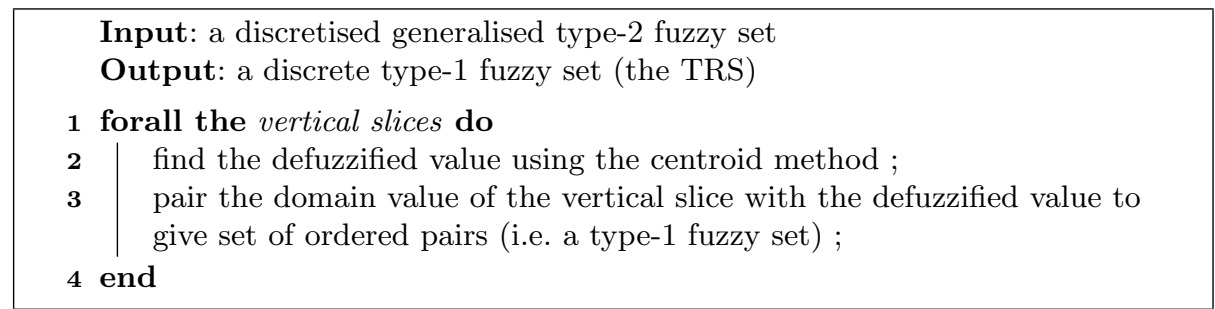

Algorithm 2: VSCTR of a discretised type-2 fuzzy set to a type-1 fuzzy set.

In 10, VSCTR performed well for both efficiency and accuracy when compared experimentally with other generalised type- 2 defuzzification techniques against benchmark values generated by exhaustive defuzzification. The experiments reported in [9] demonstrate that the Nie-Tan defuzzified value (of the interval type-2 fuzzy set) approximates to the exhaustive defuzzified value more closely as domain discretisation becomes finer.

\subsection{The $\alpha$-Plane Representation}

Another recognised technique for the defuzzification of generalised type-2 fuzzy sets employs the $\alpha$-Planes Representation, proposed by Liu in 2008, 21, 25.6. In this strategy a generalised type-2 fuzzy set is decomposed into a set of $\alpha$ planes, which are horizontal slices equivalent to interval type-2 fuzzy sets. Each $\alpha$-plane is then defuzzified via the Karnik-Mendel Iterative Procedure (KMIP) [21], so forming an approximation to the TRS. Defuzzifying the resultant type-1 fuzzy set gives a defuzzified value for the generalised type-2 fuzzy set. Below this method is presented algorithmically (Algorithm 3), and diagrammatically (Fig. 3 .

Though the $\alpha$-Planes Method was envisaged by Liu as being used in conjunction with the KMIP 21, any interval defuzzification method may be used. Any variation on the KMIP, such as the Enhanced Iterative Algorithm with Stop Condition (EIASC) 28] will locate the endpoints of the TRS interval. Other interval methods, such as the Greenfield-Chiclana Collapsing Defuzzifier [12, 13], or the Nie-Tan Method [26], will defuzzify the $\alpha$-plane [8]; their defuzzified val-

\footnotetext{
${ }^{6}$ Independently of Liu, and at about the same time, Wagner and Hagras introduced the notion of zSlices [27, a concept very similar to that of $\alpha$-planes
} 


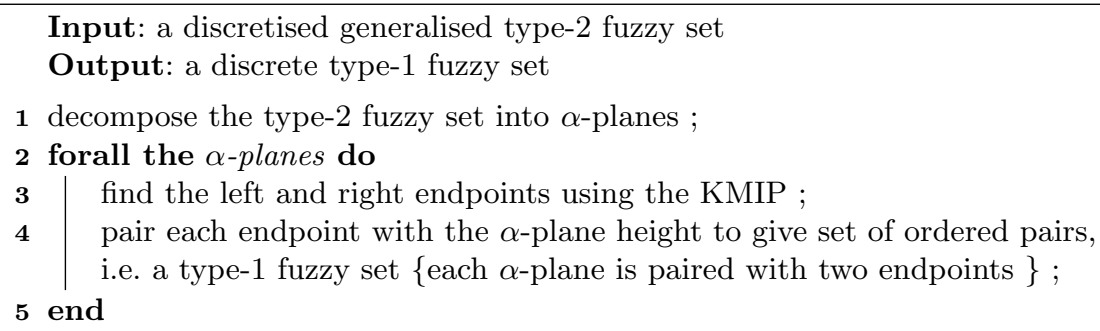

Algorithm 3: Type-reduction of a type-2 fuzzy set to a type-1 fuzzy set using the $\alpha$-Plane Method.

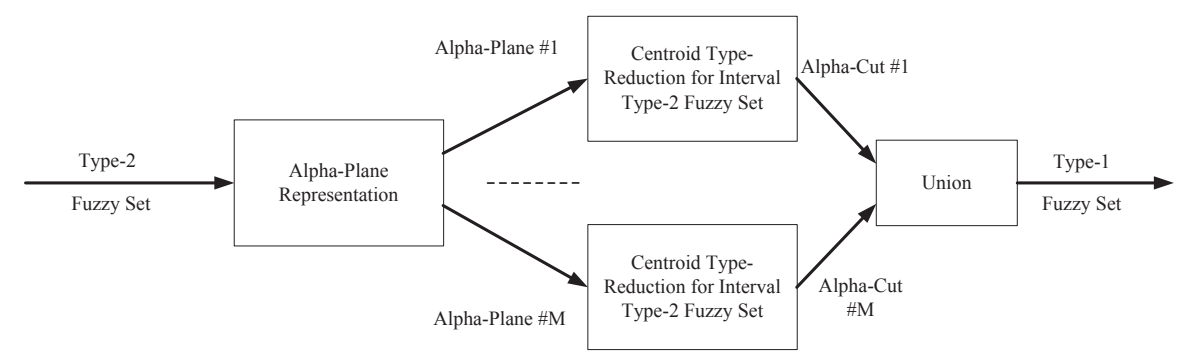

Fig. 3. Type-reduction using the $\alpha$-Planes Representation (from Liu [21]).

ues (located in the vicinity of the centre of the interval) may then be formed into a type-1 fuzzy set equivalent to the TRS.

In [10] the $\alpha$-Planes Method has been shown to be inferior to two generalised defuzzification techniques, the Sampling Defuzzifier 14 and Vertical Slice Centroid Type Reduction (VSCTR) 22, in relation to both accuracy and efficiency. The concept of the truncated generalised type-2 fuzzy set is introduced in 7, where it is shown that applying the $\alpha$-planes strategy to the truncated type- 2 fuzzy set makes for more efficient defuzzification, since there are fewer $\alpha$-planes to process. Intuitive, one might expect that accuracy would also be improved, as irrelevant $\alpha$-planes (between the maximum secondary membership grade and the truncation grade) would be eliminated and therefore not be able to distort the defuzzified value. However experiments show this not to be the case; in 22 out of 25 instances truncation worsens accuracy [7. This points to deeply entrenched issues with the method's accuracy.

\section{Conclusion}

Generalised type-2 fuzzy sets may be defined through vertical slices, or equivalently, through wavy slices. The join and meet algorithms which drive the fuzzification and inferencing stages of the FIS are always implemented via vertical 
slices. Regarding defuzzification, approaches have been derived from each of the three slicing techniques. Exhaustive defuzzification, based on the wavy slice representation, is absolutely precise but prohibitively inefficient. VSCTR has been shown experimentally to provide an excellent approximation to the exhaustive method, and to be the fastest of the three techniques. Experiments have shown the $\alpha$-Planes Method to be inferior to VSCTR as regards both speed and accuracy [10].

\subsection{Further Work}

In order to exploit the generalised type-2 fuzzy set's particular ability for uncertainty handling, more generalised type- 2 applications need to be created. This requires the development of optimised algorithms to overcome the problem of computational complexity in generalised type- 2 fuzzy inferencing. The research reported in [10, 15] shows the progress already made towards this objective. However further efficiencies are feasible in both inferencing and defuzzification.

ExpressJAM: The FastJAM (Fast Join and Meet) optimisation [15] reduces computational complexity in the FIS inferencing stages. Initial work has begun on ExpressJAM (Express Join and Meet), a further optimisation of FastJAM that applies to the particularly complex aggregation substage of the inferencing stage, and is optimisable yet further in software via parallel processing.

Generalised Greenfield-Chiclana Collapsing Defuzzifier: The GreenfieldChiclana Collapsing Defuzzifier is an interval type-2 method whose superiority over other interval methods is demonstrated in [10]. Generalisation of this interval technique to the generalised type-2 fuzzy set will result in the Generalised Greenfield-Chiclana Collapsing Defuzzifier. 


\section{Bibliography}

[1] Abbadi, A., Nezli, L., Boukhetala, D.: A nonlinear voltage controller based on interval type 2 fuzzy logic control system for multimachine power systems. International Journal of Electrical Power and Energy Systems 45(1), $456-467$ (2013)

[2] Castillo, O., Melin, P.: A Review on the Design and Optimization of Interval Type-2 Fuzzy Controllers. Applied Soft Computing 12, 1267 - 1278 (2012), DOI: http://dx.doi.org/10.1016/j.asoc.2011.12.010

[3] Castillo, O., Melin, P.: A review on interval type-2 fuzzy logic applications in intelligent control. Information Sciences 279, 615 - 631 (2014)

[4] Celik, E., Bilisik, O.N., Erdogan, M., Gumus, A.T., Baracli, H.: An integrated novel interval type-2 fuzzy MCDM method to improve customer satisfaction in public transportation for Istanbul. Transportation Research Part E: Logistics and Transportation Review 58, 28 - 51 (2013)

[5] Dereli, T., Altun, K.: Technology evaluation through the use of interval type-2 fuzzy sets and systems. Computers and Industrial Engineering 65(4), $624-633(2013)$

[6] Esposito, M., Pietro, G.D.: Interval type-2 fuzzy logic for encoding clinical practice guidelines. Knowledge-Based Systems 54, 329 - 341 (2013), DOI: http://dx.doi.org/10.1016/j.knosys.2013.10.001

[7] Greenfield, S., Chiclana, F.: Type-Reduced Set Structure and the Truncated Type-2 Fuzzy Set. Under review for IEEE Transactions on Fuzzy Systems.

[8] Greenfield, S., Chiclana, F.: Combining the $\alpha$-Plane Representation with an Interval Defuzzification Method. In: Proceedings of EUSFLAT-LFA 2011. pp. 920-927. Aix-les-Bains, France (July 2011)

[9] Greenfield, S., Chiclana, F.: Type-Reduction of the Discretised Interval Type-2 Fuzzy Set: Approaching the Continuous Case through Progressively Finer Discretisation. Journal of Artificial Intelligence and Soft Computing Research 1, 183 - 193 (2011)

[10] Greenfield, S., Chiclana, F.: Accuracy and Complexity Evaluation of Defuzzification Strategies for The Discretised Interval Type-2 Fuzzy Set. International Journal of Approximate Reasoning 54(8), 1013 - 1033 (October 2013), DOI: http://dx.doi.org/10.1016/j.ijar.2013.04.013

[11] Greenfield, S., Chiclana, F.: Defuzzification of the Discretised Generalised Type-2 Fuzzy Set: Experimental Evaluation. Information Sciences 244, 1 25 (September 2013), DOI: http://dx.doi.org/10.1016/j.ins.2013.04.032

[12] Greenfield, S., Chiclana, F., Coupland, S., John, R.I.: The Collapsing Method of Defuzzification for Discretised Interval Type-2 Fuzzy Sets. Information Sciences 179(13), 2055-2069 (June 2009), DOI: http://dx.doi.org/10.1016/j.ins.2008.07.011

[13] Greenfield, S., Chiclana, F., John, R.I.: Type-Reduction of the Discretised Interval Type-2 Fuzzy Set. In: Proceedings of FUZZ-IEEE 2009. pp. 738743. Jeju Island, Korea (August 2009) 
[14] Greenfield, S., Chiclana, F., John, R.I., Coupland, S.: The Sampling Method of Defuzzification for Type-2 Fuzzy Sets: Experimental Evaluation. Information Sciences 189, 77 - 92 (April 2012), DOI: http://dx.doi.org/10.1016/j.ins.2011.11.042

[15] Greenfield, S., John, R.I.: Optimised Generalised Type-2 Join and Meet Operations. In: Proc. FUZZ-IEEE 2007. pp. 141-146. London (July 2007)

[16] Greenfield, S., John, R.I.: The Uncertainty Associated with a Type-2 Fuzzy Set. In: Rudolf Seising (editor) Views on Fuzzy Sets and Systems from Different Perspectives, in 'Studies in Fuzziness and Soft Computing', series editor Janusz Kacprzyk. vol. 243, pp. 471-483. Springer-Verlag (2009), DOI: http://dx.doi.org/10.1007/978-3-540-93802-6_23

[17] John, R.I.: Perception Modelling using Type-2 Fuzzy Sets. Ph.D. thesis, De Montfort University (2000)

[18] John, R.I., Coupland, S.: Type-2 Fuzzy Logic: A Historical View. IEEE Computational Intelligence Magazine 2(1), 57 - 62 (February 2007), DOI: 10.1109/MCI.2007.357194

[19] Leekwijck, W.V., Kerre, E.E.: Defuzzification: Criteria and Classification. Fuzzy Sets and Systems 108, 159 - 178 (1999), DOI: 10.1016/j.fss.2008.06.018

[20] Linda, O., Manic, M.: General Type-2 Fuzzy C-Means Algorithm for Uncertain Fuzzy Clustering. IEEE Transactions on Fuzzy Systems 20(5), 883 - 897 (October 2012), DOI: 10.1109/TFUZZ.2012.2187453

[21] Liu, F.: An Efficient Centroid Type-Reduction Strategy for General Type-2 Fuzzy Logic System. Information Sciences 178(9), 2224-2236 (2008), DOI: 10.1016/j.ins.2007.11.014

[22] Lucas, L.A., Centeno, T.M., Delgado, M.R.: General Type-2 Fuzzy Inference Systems: Analysis, Design and Computational Aspects. In: Proceedings of FUZZ-IEEE 2007. pp. 1743-1747. London (2007)

[23] Mendel, J.M.: Uncertain Rule-Based Fuzzy Logic Systems: Introduction and New Directions. Prentice-Hall PTR (2001)

[24] Mendel, J.M., John, R.I.: Type-2 fuzzy sets made simple. IEEE Transactions on Fuzzy Systems 10(2), 117 - 127 (2002), DOI: http://dx.doi.org/10.1109/91.995115

[25] Mendel, J.M., Liu, F., Zhai, D.: $\alpha$-Plane Representation for Type-2 Fuzzy Sets: Theory and applications. IEEE Transactions on Fuzzy Systems 17(5), 1189 - 1207 (2009)

[26] Nie, M., Tan, W.W.: Towards an Efficient Type-Reduction Method for Interval Type-2 Fuzzy Logic Systems. In: Proceedings of FUZZ-IEEE 2008. pp. 1425 - 1432. Hong Kong (June 2008)

[27] Wagner, C., Hagras, H.: Toward General Type-2 Fuzzy Logic Systems based on zSlices. IEEE Transactions on Fuzzy Systems 18(4), 637 - 660 (2010)

[28] Wu, D., Nie, M.: Comparison and Practical Implementation of TypeReduction Algorithms for Type-2 Fuzzy Sets and Systems. In: Proceedings of FUZZ-IEEE 2011. pp. 2131 - 2138. Taiwan (2011)

[29] Zadeh, L.A.: Fuzzy Sets. Information and Control 8, 338 - 353 (1965) 
[30] Zadeh, L.A.: The Concept of a Linguistic Variable and its Application to Approximate Reasoning. Information Sciences 8, 199 - 249 (1975)

[31] Zadeh, L.A.: The Concept of a Linguistic Variable and its Application to Approximate Reasoning - II. Information Sciences 8, 301 - 357 (1975)

[32] Zadeh, L.A.: The Concept of a Linguistic Variable and its Application to Approximate Reasoning - III. Information Sciences 9, 43 - 80 (1975)

[33] Zhou, S.M., Chiclana, F., John, R.I., Garibaldi, J.M.: Type-1 OWA Operators for Aggregating Uncertain Information with Uncertain Weights Induced By Type-2 Linguistic Quantifiers. Fuzzy Sets and Systems (ISSN 0165-0114) 159(24), 3281 - 3296 (December 2008), DOI: http://dx.doi.org/10.1016/j.fss.2008.06.018 